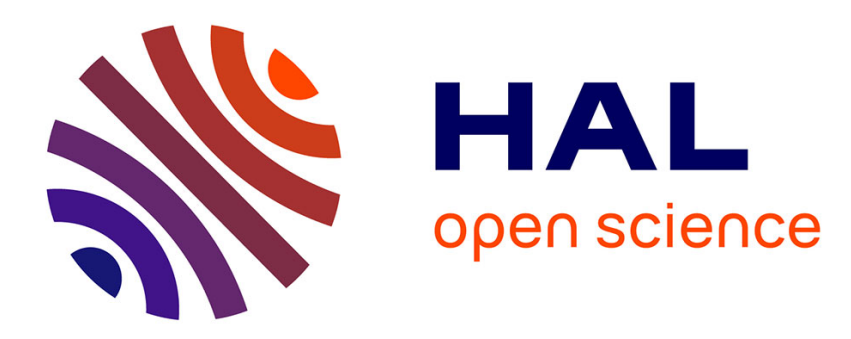

\title{
New methods for the geometrical analysis of tubular organs
}

Florent Grélard, Fabien Baldacci, Anne Vialard, Jean-Philippe Domenger

\section{To cite this version:}

Florent Grélard, Fabien Baldacci, Anne Vialard, Jean-Philippe Domenger. New methods for the geometrical analysis of tubular organs. Medical Image Analysis, 2017, 42, pp.89 - 101. 10.1016/j.media.2017.07.008 . hal-01618611

\section{HAL Id: hal-01618611 \\ https://hal.science/hal-01618611}

Submitted on 18 Oct 2017

HAL is a multi-disciplinary open access archive for the deposit and dissemination of scientific research documents, whether they are published or not. The documents may come from teaching and research institutions in France or abroad, or from public or private research centers.
L'archive ouverte pluridisciplinaire $\mathbf{H A L}$, est destinée au dépôt et à la diffusion de documents scientifiques de niveau recherche, publiés ou non, émanant des établissements d'enseignement et de recherche français ou étrangers, des laboratoires publics ou privés. 


\title{
New methods for the geometrical analysis of tubular organs
}

\author{
Florent Grélard ${ }^{\mathrm{a}, \mathrm{b}, *}$, Fabien Baldacci $^{\mathrm{a}, \mathrm{b}}$, Anne Vialard ${ }^{\mathrm{a}, \mathrm{b}}$, Jean-Philippe Domenger $^{\mathrm{a}, \mathrm{b}}$ \\ ${ }^{a}$ Univ. Bordeaux, LaBRI, UMR 5800, F-33400 Talence, France \\ ${ }^{b}$ CNRS, LaBRI, UMR 5800, F-33400 Talence, France
}

\begin{abstract}
This paper introduces new methods to study the shape of tubular organs. Determining precise cross-sections is of major importance to perform geometrical measurements, such as diameter, wall-thickness estimation or area measurement. Our first contribution is a robust method to estimate orthogonal planes based on the Voronoi Covariance Measure. Our method is not relying on a curve-skeleton computation beforehand. This means our orthogonal plane estimator can be used either on the skeleton or on the volume. Another important step towards tubular organ characterization is achieved through curve-skeletonization, as skeletons allow to compare two tubular organs, and to perform virtual endoscopy. Our second contribution is dedicated to correcting common defects of the skeleton by new pruning and recentering methods. Finally, we propose a new method for curve-skeleton extraction.
\end{abstract}

Keywords: Tubular organ analysis, Orthogonal planes, Centerlines, Voronoï covariance measure.

\section{Introduction}

Tubular organ analysis is essential for the physiological understanding of this kind of organs and for the characterization of related diseases. Many major diseases involve tubular organs. For instance, in the case of airway-trees, chronic obstructive pulmonary disease (COPD) has been reported to be one of the major causes of death (Murray and Lopez, 1996). Another example is coronary heart disease, which is linked to obstructed vessels, and is the first cause of death in the US (Members et al. 2008). On another note, images of neurons acquired from laser-scanning microscopy help building the relationship between their morphology and their function (Dima, 2002). All the above-mentioned organs can be considered tubular, that is to say they are $3 \mathrm{D}$ objects with a circular or elliptical crosssection and elongation in one direction.

A lot of work and recent improvements have been made to segmentation techniques regarding airwaytrees (Lo et al. , 2010) and to neuron reconstruction (Janoos et al., 2009). However, various charac-

\footnotetext{
* Corresponding author

Email address: florent.grelard@labri.fr (Florent Grélard)
}

Preprint submitted to Medical Image Analysis terization approaches still have defects with respect to tubular organs, as explained in Section 2 .

The study of tubular organs relies on measurements such as lumen area, wall thickness, and diameter estimation, and has applications such as virtual endoscopy and shape matching. Measurements on tubular organs are precise only if they are taken from a cross-section orthogonal to the tube. The usual approach to obtain orthogonal planes consists in computing the curve-skeleton (or centerline). The cross-sectional plane is defined by the tangent at a given skeletal point on the skeleton. Precise measurements can then be performed in the computed cross-section. Thus, orthogonal plane estimation is central but is usually heavily reliant on the properties of the curve-skeleton.

Curve-skeletons, or centerlines, are simplifications of shapes consisting in a set of curves. They allow to make geometrical measurements such as tangent estimation (Postolski et al., 2012) or tortuosity (Lang et al., 2012), and to perform virtual endoscopy (Bauer and Bischof, 2008). However, orthogonal plane estimation and skeleton computation are impeded due to the organ's variability in diameter, to the presence of junctions (one tube divides in two or more) and to segmentation issues. Curve-skeletons can contain irregularities 


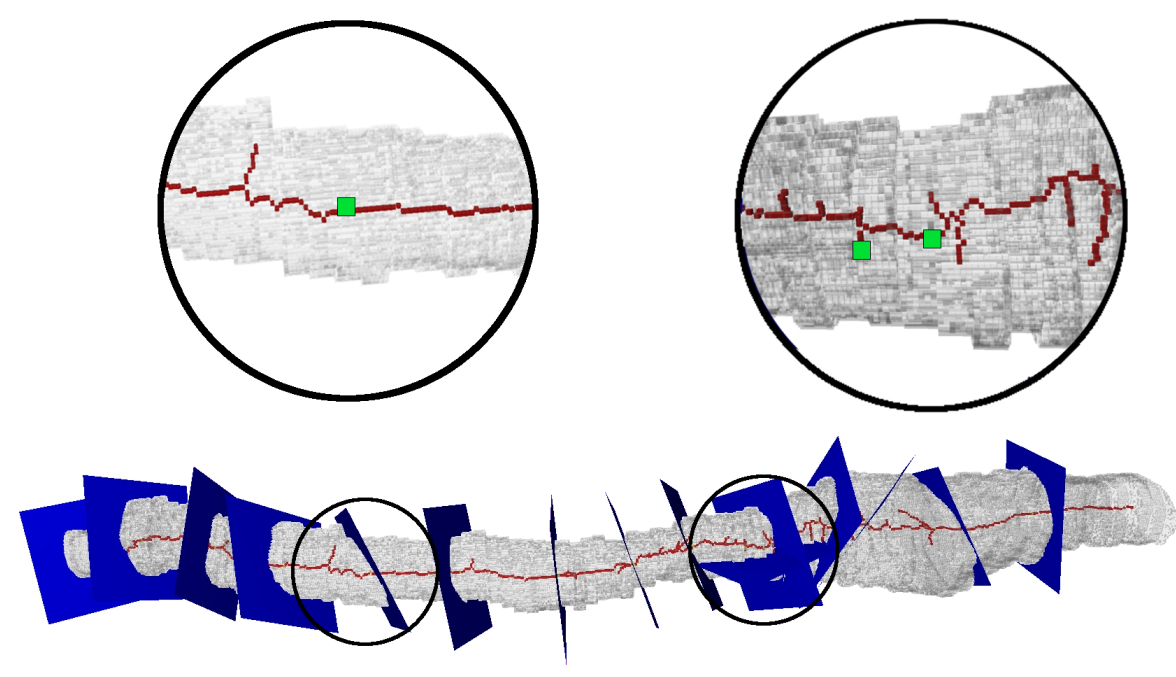

Figure 1: Neurite volume from the SNEMI3D dataset (Kasthuri et al. 2015) (in gray) and skeleton computed by the method in Lee et al. (1994) (in red). Orthogonal planes are estimated using the $\lambda$-MST method of Postolski et al. (2012) (in blue). The skeleton contains irregularities and spurious branches (see green points in the close-ups) which results in a deviated estimated tangent. As a result, orthogonal plane estimation is impacted.

(i.e. they are not smooth) which impact orthogonal plane computation (see Fig. 1). Moreover, spurious branches appear as a result of small irregularities on the object's surface which are kept through the skeletonization process. The orthogonal planes computed on faulty branches are not orthogonal to the tube. Thus, filtering the skeleton is often a necessary post-processing step. Pruning consists in the removal of irrelevant parts of the skeleton, yielding a skeleton representing the original shape more aptly. Once all the extra branches have been removed, there is a one-to-one correspondence between the parts of the skeleton and those of the volume, which allows to analyze the organ further. One of the main challenges of pruning approaches is having a complete and sensitive method, meaning a method which removes all spurious branches while keeping meaningful ones. This problem arises specifically for varying-diameter tubes, where the geometry of spurious branches may vary depending on the tube radius.

Once the spurious branches have been removed, it is possible to analyze the organ more easily. Shape decomposition is part of this process. It consists in breaking a complex shape into smaller and meaningful parts. In the case of the airway-tree, each bronchus is separated from another. Nevertheless, obtaining clear delineations in lower diameter tubes is not easily achievable.

Recentering is also key to filtering the skeleton. Indeed, not all skeletons are centered or smooth inside the shape, negatively impacting geometrical analyses and virtual endoscopy. Thus, for instance, a tangent vector computed at a non-centered skeleton point is potentially not aligned with the tube's axis.

The contribution of this paper is manifold. First, we introduce a robust orthogonal plane estimator. Our method is based on the Voronoi Covariance Measure (VCM), Mérigot et al. (2011). This measure describes the shape of the Voronoi cells generated by the skeleton points or directly by the volume points. In the following section, we show how Voronoi cells have a simple and intuitive connection to orthogonal plane normals. The main advantage of our approach is its robustness to small irregularities in the curve. Moreover, unlike most orthogonal plane estimators, it does not necessarily rely on a curve, but it can be computed directly from a tubular volume.

Second, we propose original algorithms to improve existing curve-skeletons. We use the orthogonal planes to filter existing skeletons, which involves pruning and recentering steps.

Finally, we propose our own curveskeletonization approach. Our algorithm consists 
in a tracking procedure, which direction is given by the orthogonal planes. It consists in two steps: the first one is dedicated to tubular parts, the second one to junctions. We show our skeleton is complete and possesses few to no faulty branches.

The paper is organized as follows. In Section 2 . we describe the state-of-the-art methods for curveskeleton extraction, and orthogonal plane estimation. Section 3 presents our method for robust orthogonal plane estimation. In Sections 4 and 5 existing skeletons are filtered and improved using orthogonal planes. In Section 6, we introduce our own centerline tracking algorithm.

\section{Related work}

The most common workflow to obtain robust measurements on tubular organs consists in: segmenting the organ; extracting the curve-skeleton from the volume; computing cross-sections from the curve-skeleton; and making measurements from the cross-sections. A workflow for airway-tree analysis following these four steps is described in Tschirren et al. (2005). This section presents previous works aiming at extracting and filtering curve-skeletons and at estimating orthogonal planes.

There are various existing algorithms for curveskeleton extraction. Tagliasacchi et al. (2016) described a list of desirable properties for a curveskeleton to possess. In the context of tubular organs, not all properties are necessary. The curveskeleton must be complete, centered, thin and not impacted by noise. The curve-skeleton can be extracted directly from a gray-level image, but an implicit segmentation step is usually involved to capture the geometry of the organ (Flasque et al. 2001).

Thus, in the context of varying-diameter tubes with junctions, we focus on curve-skeleton methods based on segmented sets of voxels. According to the survey by Cornea et al. (2007) we can classify the curve-skeleton algorithms for discrete objects in the following classes: (a) thinning algorithms and (b) distance field or general field based algorithms.

Thinning methods consist in the removal of voxels from the boundary until a thin version of the shape is obtained. They are comprised, among many others, of the work of Lee et al. (1994), Palágyi et al. (2006) and Couprie et al. (2007) (see Fig. 2). The first two compute the skeleton on any given $3 \mathrm{D}$ object whereas the latter is applied to tubular shapes. The authors in Couprie et al. (2007) propose to compute the skeleton by filtering the medial axis (the centers of all the local maximal balls that can fit inside a shape) with an angle criterion. The resulting filtered medial axis is used as a constraint set for the computation of the skeleton by homotopic thinning. More importantly, and this is true for all thinning methods, it can create small faulty branches (see close-up in Fig. 2a), often removed automatically by a pruning step, which are, in our case, difficult to distinguish from small branches that are representative of the volume. The authors define two parameters in order to get rid of spurious branches. However, in practice, some small spurious branches remain, regardless of the parameter values.

Potential field methods generally produce very smooth skeletons. For instance, we can cite Cornea et al. (2005), Hassouna and Farag (2009) where the skeleton is extracted from a potential field computed on the volume. In Cornea et al. (2005), the authors compute a hierarchical skeleton based on a Newtonian potential field. Each boundary point is considered as an electric charge, repelling interior points of the volume. From the potential vector field, critical points (points where the magnitude of the force vector vanishes) are extracted and connected thanks to paths, yielding a first hierarchy in the skeleton. Then, two levels of hierarchy are added through high divergence value and highcurvature points. The main drawback of this kind of algorithm is that the resulting skeleton is not necessarily connected, can be incomplete (see Fig. 2b and can contain spurious branches (see Fig. 2c).

In summary, the various classes of curve-skeleton algorithms have defects, including introducing irregularities, being incomplete, or having faulty branches. Pruning consists in removing faulty branches while keeping meaningful ones with respect to the object. The general idea is to define a significance measure for each point: this value corresponds to the relevance of a point in the skeleton, and is preferably low for spurious branches. Various significance measures have been defined and reviewed in Shaked and Bruckstein (1998). These measures aim at determining if a branch stems from a small irregularity on the surface. For instance, maximal thickness of implied erosion (Arcelli and Sanniti di Baja, 1993) estimates the part of a volume which is encoded by the branch. Another measure is dedicated to the ratio between the length of the branch and the number of closest boundary points to a branch point. A recent work intro- 


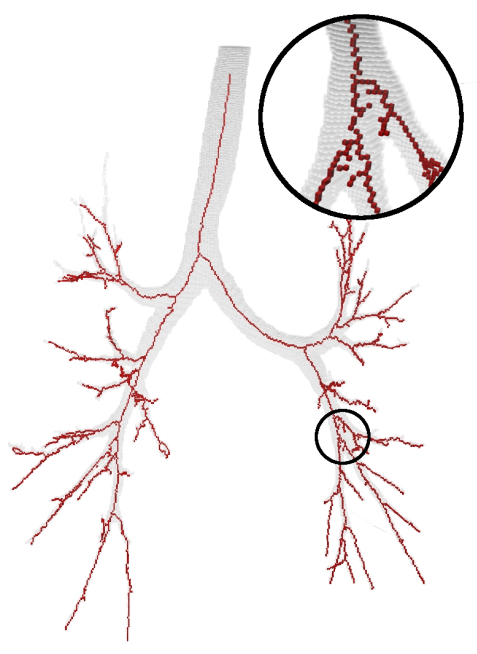

(a)

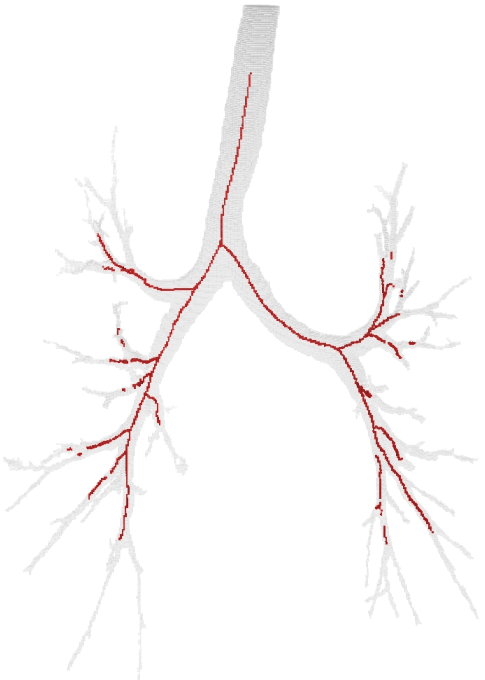

(b)

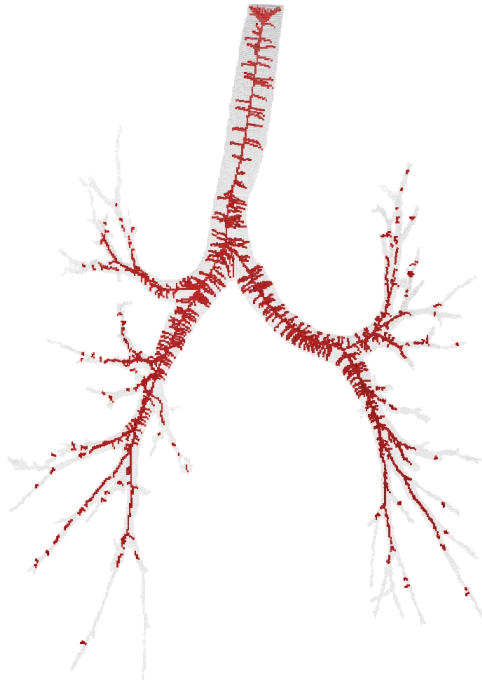

(c)

Figure 2: (a) Skeleton (in red) computed on a airway-tree segmentation using the thinning method described in Couprie et al. (2007): parameter values were chosen to obtain the most complete skeleton with the least amount of faulty branches, but there are some regardless, see close-up view. (b) (c) Skeleton computed using potential field (Cornea et al., 2005): two levels of hierarchy on a skeleton obtained with two different parameter values. Obtaining a complete skeleton containing all the branches in the volume as well as no faulty branches is not possible.

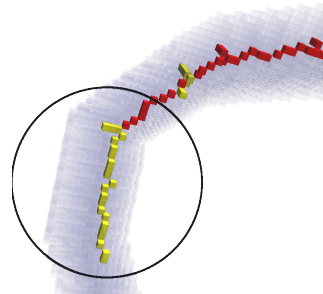

(a)

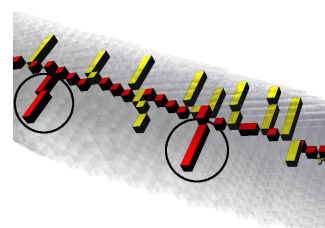

(b)
Figure 3: Pruned branches (in yellow) of a skeleton computed using Cornea et al. (2005) on tubular volumes (in gray). The pruning method is described in Serino and Sanniti di Baja (2014). Their centeredness criterion leads to errors (see circled areas): (a) some relevant branches are pruned and (b) some spurious branches are kept.

duced in Serino and Sanniti di Baja $(2014)$ involves pruning 3D-curve skeletons. It considers the curveskeleton as a graph, where each branch is an edge. Pruning is done in a hierarchical manner. As a first step, all peripheral edges are examined. Then, a neighboring internal edge can be pruned only if all of its neighboring peripheral edges have been deleted. The authors use the combination of four significance measures to achieve pruning: a length criterion, a criterion estimating whether the branch is centered, and two criteria involving the amount of information the branch encodes in the shape. The thresholds for each criterion are set automatically by averaging measures over branches. This method has two main drawbacks with respect to our tubeshaped volumes. First, one criterion is relying on centeredness of the branch. However, certain algorithms cannot guarantee this property (see Fig. 3 ). Second, using the average of the significance measure might not be relevant in the context of varyingdiameter shapes.

Another post-processing step consists in recentering the skeleton. Points are not necessarily centered in the branch (see Fig. 3a), or branching points differ from one method to another (see Sobiecki et al. (2014) for example). Zigzags in the skeleton impact geometrical measurements leading, for example, to an overestimation of the length. Bradley and Withers (2016) smooth and recenter skeletons as a post-processing step. First, they compute the set of maximal balls centered at each point of the input skeleton. Then, they compute the set of minimally overlapping balls. The resulting centered and smooth skeleton consists in the centers of the balls linked by splines. Although the presented approach is interesting to make measurements in the context of noisy data, it can discard interesting small geometrical features as well. Bar- 


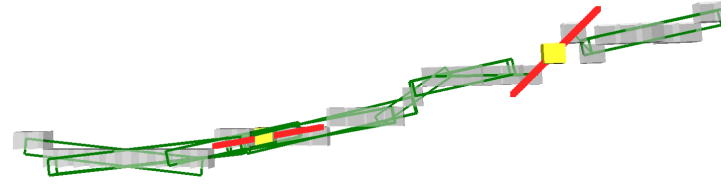

Figure 4: Tangent estimation (in red) at two curve points (in yellow) using the 3D $\lambda$-MST method (Postolski et al. 2012). The tangent is given as a weighted average of the digital straight segments (in green). Due to irregularities on the curve, the tangent estimated at the point on the right does not correspond to the shape of the curve.

bieri et al. (2015) describe an approach to recenter a skeleton inside a shape. The new recentered point correspond to a centroid in the cross-section. The main drawback of this method is the orthogonal plane estimation is very sensitive to irregularities in the skeleton, and junctions are not properly processed.

Orthogonal plane estimation is usually based on the skeleton. The tangent at a point on the curve-skeleton is the normal of the orthogonal plane. Tangent estimation at a point $p$ on a 3D discrete curve can be achieved in a naive way, by computing the vector defined by the difference between two points in the skeleton. The $\lambda$-MST estimator described in Postolski et al. (2012) allows to estimate 3D tangent more robustly. It relies on maximal segments (longest digital straight segments) which capture the shape of the curve. The tangent at $p$ corresponds to a weighted average of the direction vectors of all maximal segments passing through $p$ (see Fig. 4). Both the naive and the $\lambda$-MST methods are insufficient as they are sensitive to irregularities in the discrete curve. Other methods such as Kang et al. (2006) use non-rigid registration of a deformable cylinder on the acquisition image, and compute orthogonal planes from the axis of this cylinder. However, the fitted cylinder has a constant diameter. Thus, this method is not suited for varying-diameter tubes.

This paper addresses the shortcomings of the afore-mentioned methods, and proposes a new orthogonal plane estimator which is more accurate than existing methods and does not necessarily rely on a curve-skeleton computation for certain types of tubular volumes. Moreover, we introduce automatic post-processing approaches for the curveskeleton, including pruning and recentering which tackle the issues mentioned above. Finally, we present a new algorithm for curve-skeleton extrac- tion on tubular volumes which yields complete and centered skeletons.

\section{Orthogonal plane estimation}

\subsection{Voronoi Covariance Measure}

All of our contributions are based on the Voronoi Covariance Measure (VCM). It is a measure, first introduced in Mérigot et al. (2011) on point clouds which was used to estimate normals. The VCM was also defined on sets of voxels in Cuel et al. (2014) and was proven to be a reliable tool to estimate the surface normal. This measure was shown to be resilient to Hausdorff noise and to outliers (Cuel et al. 2015).

Let $y$ be a point in a digital set. The VCM considers the covariance matrix of Voronoi cells in the neighborhood of $y$. The eigenvector with the largest eigenvalue in this matrix corresponds to the surface normal. Since the Voronoi diagram is global by nature and sensitive to noise and digitization effects, VCM computation is restricted to a small computation window taking into account a local neighborhood. This ensures robustness of the estimator and its multigrid convergence properties (Cuel et al. 2014).

We recall here the formal definition of the VCM. In the following, we refer to digital points, i.e. centers of pixels in $2 \mathrm{D}$ or voxels in $3 \mathrm{D}$, as points. Let us denote by $n$ the dimension, $O \subseteq \mathbb{Z}^{n}$ the object, $B(o, \rho)$ the points in the ball of radius $\rho$ and centered at $o$. The $R$-offset of $O$ is defined as $O^{R}=\cup_{o \in O} B(o, R)$. The $R$-offset bounds the Voronoi cells $\operatorname{Vor}($.$) , allowing to extract local infor-$ mation from the Voronoi diagram. Voronoi cells in a neighborhood around a point are taken into account: at a given point $y$, the computation window is defined as $W=B(y, r) \cap O$, where $r$ is called integration radius. The domain of integration for the $\mathrm{VCM}$ at $y$ is given as:

$$
D I_{O}(y, r, R)=\cup_{w \in W}\left(\operatorname{Vor}(w) \cap O^{R}\right)
$$

The VCM is the covariance matrix of all vectors between the points in the domain of integration and their respective Voronoi sites (see Fig. 5a). The site for a point $x$ is denoted by $p_{O}(x)$. Finally, the covariance matrix is expressed as:

$$
\mathcal{V}_{O}(y, r, R)=\sum_{x \in D I_{O}(y, r, R)}\left(x-p_{O}(x)\right)\left(x-p_{O}(x)\right)^{T}
$$




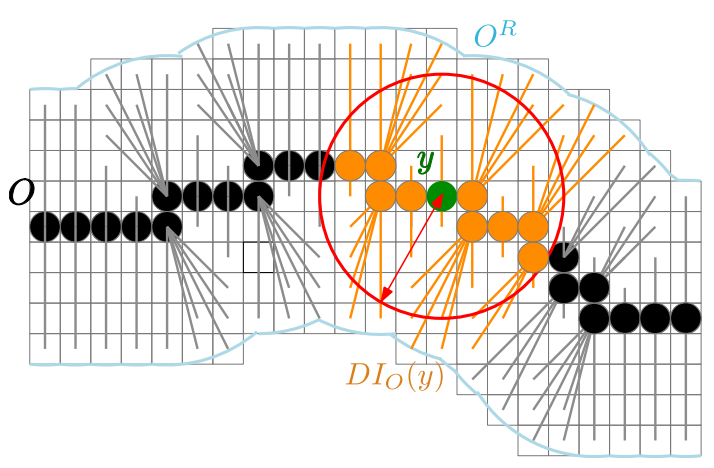

(a)

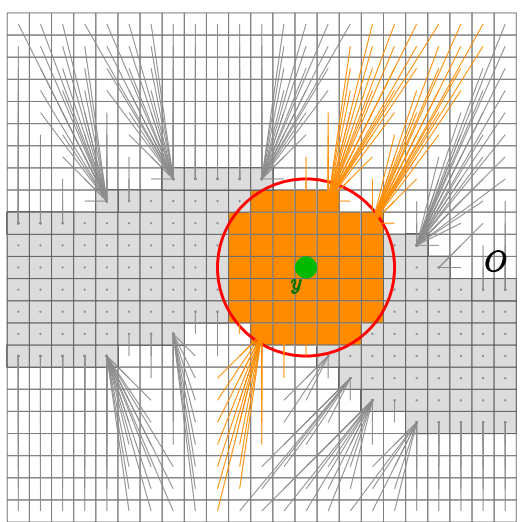

(b)

Figure 5: Digital VCM computation at point $y$ on (a) a discrete 2D curve $O$ and (b) a 2D object $O$. Solid lines (in gray and orange) show the link between points and their associated Voronoi sites. All the lines with the same Voronoi site define a Voronoi cell. (a) The VCM is the covariance measure of the Voronoi cells in the domain of integration $D I_{O}(y)$ (associated lines in orange). (b) Voronoi cells are null for points inside the object $O$, such that only border points have a Voronoi cell. Orange lines are integrated for the VCM at $y$.

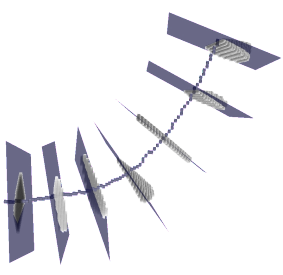

(a)

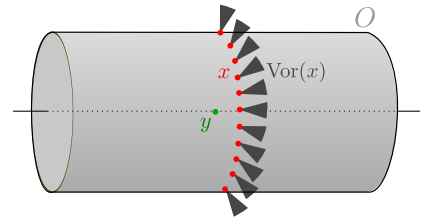

(b)
Figure 6: Correspondence between Voronoi cells and orthogonal planes. (a) Voronoi cells (in gray) computed at curve points are aligned with orthogonal planes (in blue). (b) VCM computation on a volume (a cylinder) at a point $y$. Similarly as in the $2 \mathrm{D}$ case (see Fig. 5b, only surface points $x$ have a non-null Voronoi cell $\operatorname{Vor}(x)$. The Voronoi cells $\operatorname{Vor}(x)$ are aligned with the orthogonal plane at $y$.

The VCM is defined on any compact set. Figure $5 \mathrm{a}$ shows its computation at a point on a $2 \mathrm{D}$ digital curve, and Fig. 5b shows its computation on a 2D digital object. Since the Voronoi cell of a point inside the object is reduced to the point itself, these points do not bear influence in the VCM computation. Only points on the border contribute to the VCM.

From this definition, it is clear the choice for the integration radius $r$, and $R$ is of major importance. An empirical study for the VCM parameters $r$ and $R$ has been led and its results are summarized in Section 3.3 .

\subsection{Orthogonal plane}

We propose to use the VCM to estimate orthogonal planes (as described in our work in Grélard et al. (2015)). The orthogonal plane at a given curve point is defined by its tangent to the curve. The two main elongation directions of a 3D Voronoi cell define the orientation of the orthogonal plane (see Fig.6a). As explained in Section 3.1, the VCM estimates the shape of the expected Voronoi cell at a point by integrating several digital Voronoi cells. In $2 \mathrm{D}$, the normal at a curve point is given as the largest eigenvector in the VCM. Estimating a precise orthogonal plane on a $3 \mathrm{D}$ discrete curve is the same problem as detecting a normal on a $2 \mathrm{D}$ curve point, only with a supplementary dimension. The two eigenvectors with the superior eigenvalues in the VCM give the basis for the orthogonal plane. The eigenvector with the lowest eigenvalue corresponds to the tangent to the curve.

Since the definition of the VCM holds for any compact set, the orthogonal plane estimation does not necessarily rely on a curve, and can be used directly from a set of voxels. As an example, the shape of the Voronoi cells on the surface of a cylinder are pictured in Fig. 6b. Let us denote by $y$ the point where an orthogonal plane must be estimated. There is a correspondence between the shape of the Voronoi cells at surface points close to $y$ and the expected orthogonal plane. Indeed, the union of all such Voronoi cells is aligned with the orthogonal plane. As a result, the conclusions 
drawn from a 3D discrete curve apply for a volume, and the basis of the orthogonal plane is given by the two eigenvectors with the superior eigenvalues. The main challenge in order to estimate orthogonal planes directly from the volume is to define which local points on the surface must be taken into account in the domain of integration.

\subsection{Automatic setting of the integration radius}

Let $\mathcal{P}(p)$ be an orthogonal plane at a point $p$ and $r$ the integration radius of the VCM used for the orthogonal plane computation, as described in Section 3

The integration radius $r$ must be "well-adjusted" to capture the shape of the object locally, while being robust to shape irregularities. A parameter study correlating $r$ to the radius of the tube $\rho$ can be found in an online appendix (F. Grélard et al. 2017). The results show the integration radius $r$ should be chosen as $\rho+2$. The parameter study also highlights that $r$ should be adjusted such that the domain of integration contains (a) surface points for which Voronoi cells are aligned with the expected orthogonal plane and (b) the least amount of irrelevant surface points. In the following paragraph, we use a ball as domain of integration in order to integrate all the relevant surface points, and we describe how to automatically obtain the minimal value for its radius $r$.

The size of the domain of integration is initialized with the error-free Euclidean distance transform value $(D T)$. Obviously, this value does not correspond to the expected value for $r$ in the case of a tube with elliptic cross-sections, or when the orthogonal plane is computed at a point $p$ near the boundary of the object. The process is the following: an initial orthogonal plane $\mathcal{P}_{0}(p)$ is computed with $r=D T(p)$. Let $C$ be the cross-section, i.e. the connected component in $O \cap \mathcal{P}(p)$ which contains $p$. If there is at least one point $x$ in $C$, such that $d(p, x)>r$, then $r$ is incremented. This process is repeated until no points in $C$ are found at $d>r$. It allows to converge towards both the expected orthogonal plane and the minimal value for $r$ to include all the necessary surface points in $C$.

\subsection{Results}

In this section, the VCM efficiency in computing orthogonal planes is compared to the $\lambda$-MST method. These methods have been implemented using the DGtal library (D. Coeurjolly et al. 2016)

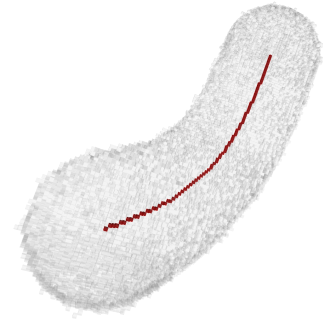

(a)

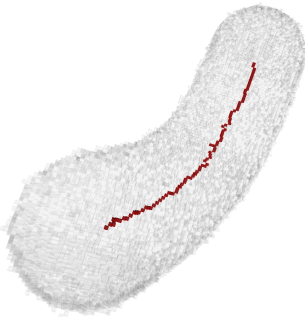

(b)
Figure 7: (a) Noisy tubular volume (in gray) generated fitting balls of constant radius at each point of the digitized initial centerline (in red). (b) Computed skeleton using the method in Lee et al. (1994) containing irregularities.

and are available along with the data online 1 . We compare the methods both on synthetic and real data.

\subsubsection{Noisy synthetic data}

The goal of this section is to compare our method to the $\lambda$-MST estimator on known volumes with altered surfaces. We have generated a slightly curved cylinder with constant diameter (see Fig. 7), and a straight elliptic cylinder with varying minor and major axis values (see Fig. 10a).

Irregularities on the surface of tubular organs are the source of skeleton distortions. In order to generate similar irregularities on the synthetic data we have added some noise on the surfaces of the synthetic object. Noisy versions of the volumes are produced using a simplified version of Kanungo's algorithm (Kanungo et al., 2000). This method adds noise on binary images by switching the value of each voxel, according to its distance $d$ to the object boundary, with a probability $\alpha^{d}$. Cavities and unconnected noisy voxels are then removed using morphological operators.

The first test to assess our method's robustness consists in extracting geometrical characteristics on the computed 2D orthogonal planes, and in comparing them to known values.

The intersection between each orthogonal plane and the unaltered digital curved cylinder should be a disk. In order to quantify how close to a disk the results are, two features are computed on the $2 \mathrm{D}$ shape resulting from the intersection: the area in number of pixels, and the roundness, given as

${ }^{1}$ https://github.com/fgrelard/ OrthogonalPlaneBasedTools 
$\frac{4 A}{\pi * a^{2}}$ where $a$ is the length of the major axis, and $A$ the area. This value ranges from 0 (line) to 1 (perfect circle).

The corresponding expected values can be computed with the known radius of the curved cylinder (20 pixels).

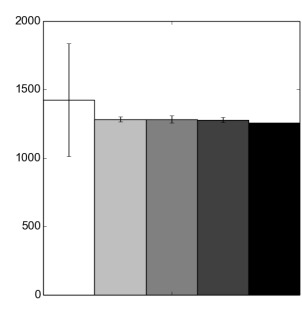

(a)

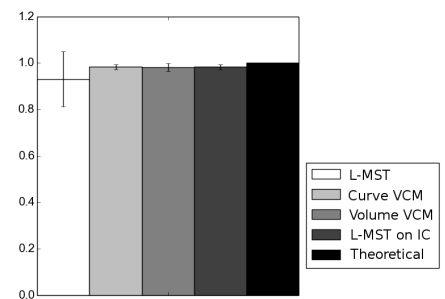

(b)
Figure 8: (a) Area in pixels and (b) roundness mean values for all the orthogonal planes found for the noisy tube volume of Fig. 7 The $\lambda$-MST on the computed skeleton (white) yields results with high-variability, whereas the $\lambda$ MST method applied on the initial centerline (L-MST on IC) and the two VCM methods applied on the computed skeleton and the volume respectively are consistent with the theoretical values.

The results are obtained on 93 cross-sections. A sample of a few orthogonal planes are shown on the curved cylinder in Fig. 9. The mean values obtained with the two variants of the VCM method (see Figs. 8a and 8b), are closer to the theoretical value than those obtained with $\lambda$-MST method on the computed skeleton. Furthermore the difference in standard-deviation between the two methods is significant. The coefficient of variation, defined as the ratio of the standard deviation to the mean, is $29 \%$ and $12 \%$ for the $\lambda$-MST, against $1.5 \%$ and $1.1 \%$ for the VCM, for the area and roundness respectively. This reflects the high-variability of the $\lambda$-MST method, as it finds a substantial number of incorrect orthogonal planes, whereas the results of the proposed method are consistent.

Another evaluation process consists in evaluating the difference between the normal direction of the estimated plane, and the known direction. This test has been performed on a straight elliptic cylinder for which the normal direction at each point of its initial centerline is constant (see Fig. 10a). For each point of the skeleton computed using the method in Lee et al. (1994), the angle defect between the computed normal and the expected normal is determined for all methods. Figure $10 \mathrm{~b}$ shows the results

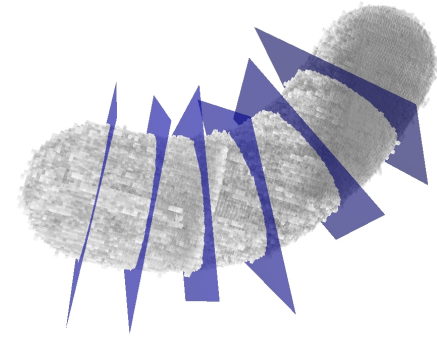

Figure 9: Examples of orthogonal planes (in blue) obtained on a noisy tube-like volume with the volumetric VCM method.

obtained on 50 slices.

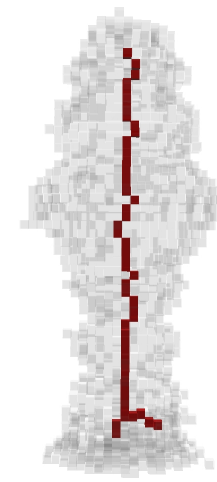

(a)

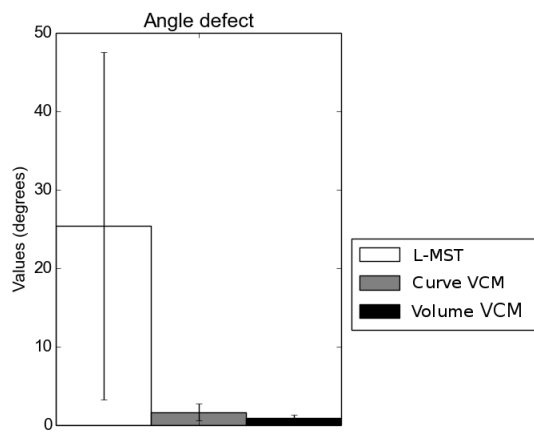

(b)
Figure 10: (a) Noisy elliptic cynlinder with varying minor and major axes, generated along a centerline with constant normal direction. The computed skeleton (Lee et al., 1994) is shown in red. (b) The angle defect (in degrees) between the computed normal and the expected normal shows our method outperforms the $\lambda$-MST tangent estimator.

Using VCM computed from both the centerline and the volume yields an angle defect close to zero, with a low standard deviation. The mean value of the angle defect are greater for the $\lambda$-MST, and again suffers from high-variability (standard deviation of 22 degrees against 1.1 and 0.46 degrees for the VCM on the curve and on the volume respectively). The $\lambda$-MST estimator does not perform well because DSS recognition is sensitive to slight pixel deviation in a curve: in some cases, short DSS are found which means the computed orientation is not representative of the actual tangent.

\subsubsection{Real data}

Estimation from the curve. We aim at evaluating our orthogonal plane estimator on real data, namely an airway-tree dataset from which a curve-skeleton 


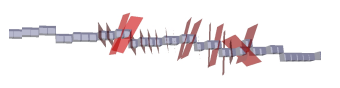

(a)

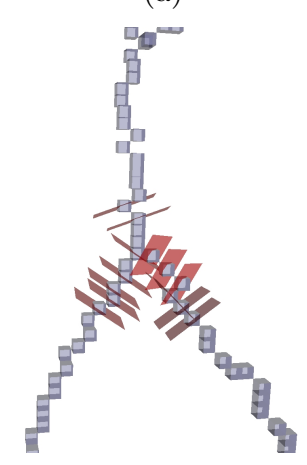

(c)

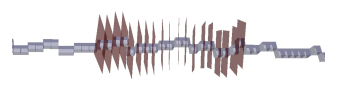

(b)

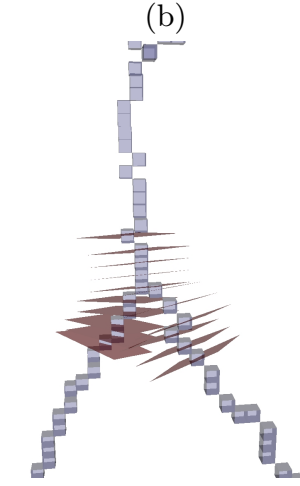

(d)
Figure 11: Orthogonal planes computed on the skeleton of an airway-tree with (a)-(c) $\lambda$-MST and (b)-(d) VCM. The orthogonal planes computed by the $\lambda$-MST method are impacted by irregularities in the curve, those computed by our method are not.

is extracted. The bronchi have been segmented manually, and the skeleton was computed on the resulting volume with the method of Lee et al. (1994). Parts of the skeleton are impacted by irregularities of the surface. This leads to some incorrect orthogonal planes estimation with the $\lambda$-MST method (see Fig. 11a) for these points. On the contrary, similarly as what was observed on synthetic data, the VCM method gives consistent results which are not affected by slight distortions in the skeleton (see Fig. 11b.

In junctions, there are various skeletal branches and this impacts the local computation. Thus, the orthogonal plane estimation is done branch by branch. Furthermore, the first planes estimated after a branching part are not of interest since not only do they contain a section from a tubular part, but they also contain a large part of the second beginning branch (see Fig. 11d).

Both results on synthetic and real data show our method is more precise than state-of-the-art algorithms to estimate orthogonal planes on a curve.

Estimation from the volume. Computation of orthogonal planes from the volume is of major interest since it does not rely on a curve-skeleton and its potential defects. Typical state-of-the-art methods are based on tangent estimation on a curve in order to estimate the orthogonal plane. Thus, only the results of our method are displayed here. The radius for the domain of integration was computed automatically according to the method described in Section 3.3 .

Estimating orthogonal planes directly from the volume requires that the input volume is tubular. The orthogonal planes may indeed not be properly estimated in parts where the volume is not locally tubular, in the sense of the domain of integration of the VCM. This is the case of the colon segmentation, in areas where the colon folds (see Fig. 12a). In such areas, the orthogonal planes may not be properly positioned. However, on tubular volumes, such as neurite volumes, the orthogonal planes are properly positioned and very close to the planes estimated from the skeleton (see Fig. 12b). Our method is robust to surface irregularities or noise located on the surface of the volume (see close-up on Fig. 12b). Regarding the airway-tree (see Fig. 12c), the planes are properly oriented in tubular parts as well.

The orthogonal plane estimation from the volume is similar to the one from the skeleton for tubular shapes. This makes the volume estimator very interesting since it is not necessary to compute the curve-skeleton as a pre-processing step. To our knowledge, it is the first orthogonal plane estimator which works directly on the volume and takes into account its geometry.

\section{Pruning}

As explained in Section 2, a lot of curveskeletonization approaches on tubular objects produce irrelevant branches originating from surface irregularities. Skeletal branches are maximal sets of connected points in the skeleton which are not branching points (i.e. points having at least three neighbors). Pruning methods aim at keeping relevant branches while removing spurious branches. Spurious branches are not aligned with the tube's axis. In this section, we propose a new significance measure based on orthogonal plane computation to estimate the deviation of a skeleton branch from the tubular object direction. This measure can be used to perform automatic pruning of an existing curve-skeleton.

\subsection{Method}

The curve-skeleton is decomposed into a set of branches, in order to decide, for each branch, 


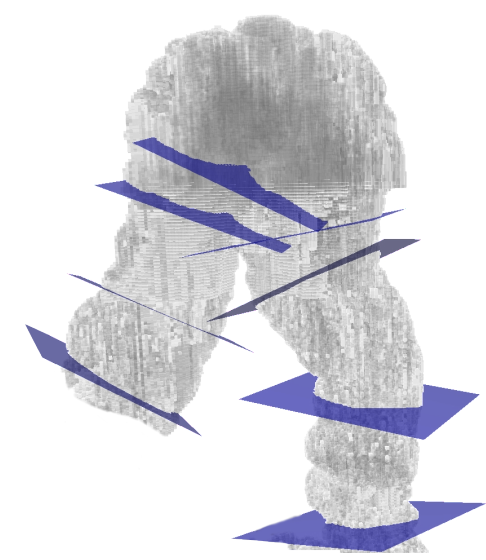

(a)

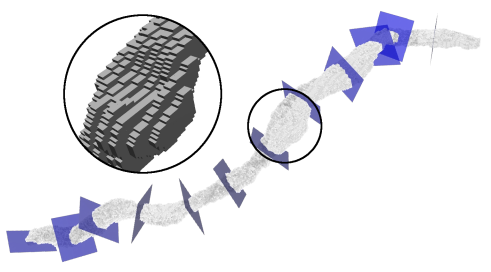

(b)

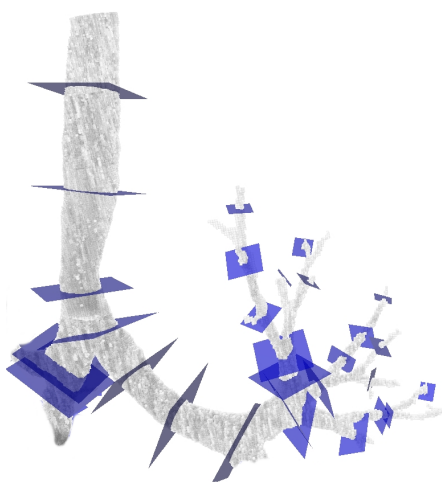

(c)

Figure 12: Orthogonal planes (in blue) computed on various tubular volumes with our method. (a) On a colon, data courtesy of Cornea et al. (2005), (b) on a neurite, data from the SNEMI3D challenge (Kasthuri et al. 2015), with a close-up showing surface irregularities: our method is not sensitive to them; and (c) on an airway-tree.

whether to keep it or prune it. Since the curveskeleton is decomposed into branches, it must satisfy the two following properties : (a) it must be thin, in order to detect branching points based on their neighborhood and (b) it must be connected. These two properties are ensured by a lot of thinning approaches, and general-field algorithms which makes it applicable on a wide variety of skeletonization methods.

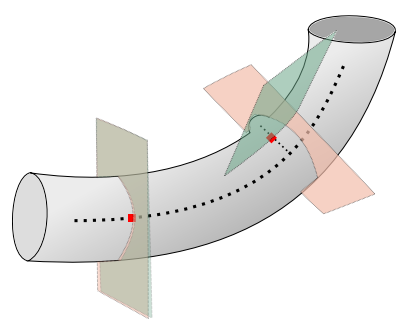

Figure 13: Difference between orthogonal planes computed from the curve (green plane) and the volume (pale red plane). On the left, the two orthogonal planes overlap, because the skeleton is aligned with the tube axis. On the right, the plane computed on a spurious branch is highly deviated compared to the one computed on the volume.

The significance measure consists in measuring the angle $\alpha$ formed by the two orthogonal planes defined, on one hand by the branch (a curve) and, on the other hand by the volume. The orthogonal plane normal of an undesired branch is directed at the object's surface, whereas its volumetric counterpart is along the shape. The angle formed by the two planes for spurious branches is large, whereas it is low for meaningful branches (see Fig. 13).
Orthogonal planes on the curve and the volume are computed with the VCM at each point in the branch (see Section 3). In order to obtain a robust estimation of the orthogonal plane on the volume, the integration radius $r$ is automatically set using the method described in Section 3.3. Regarding the computation of the orthogonal plane from the curve, $r$ depends on the branch length. Indeed, a low-resolution version of a given curve should have a lower $r$, in order to get into account only relevant local information. Experimentally, we choose $r$ as half the branch length since it gives a good representation of the tangent to the curve.

The angle difference $\alpha$ is computed for each point in the branch. The average angle difference for a branch allows to discard it or keep it according to a threshold. Unlike methods presented in Section 2 , setting a global threshold is not a problem in this case since the significance measure is independent of the local diameter of the shape. The choice for the threshold is discussed in Section 4.2

All branches in the skeleton are analyzed during the pruning procedure. Only those having a significance measure above a given threshold and preserving the same number of connected components as in the initial skeleton when removed are deleted. The skeleton is modified and updated when a branch is deleted. Computation of the significance measure is done on each branch of the updated skeleton until no branch can be removed. This ensures that the pruned skeleton is not disconnected, and that all spurious branches across the skeleton are deleted. 
Table 1: Comparison of our pruning method to the state-of-the art method in Serino and Sanniti di Baja (2014). Four measures are used to assess the completeness and sensitivity of our method compared to the state-of-the-art. The number of spurious branches deleted by the existing method and not deleted by ours (denoted by Err VCM1 $_{\text {) }}$ the number of spurious branches deleted by our method but not deleted by the existing one (ErrsT1); the number of relevant branches deleted by our method but preserved by the other (ErrVCM2); and the number of relevant branches deleted by the existing one, but preserved by ours (Errst2). These measures are computed on the two volumes of Fig. 14 and six supplementary volumes (results not shown): AW1, AW2 and AW3 (other airway-tree segmentations), PA (pelvic arteries segmentation) and N1 and N2 (neurite volumes from the SNEMI3D challenge (Kasthuri et al., 2015)).

\begin{tabular}{ccccccccc}
\hline Volumes Fig. & $14 \mathrm{a}$ & $14 \mathrm{c}$ & $\mathrm{PA}$ & $\mathrm{AW} 1$ & $\mathrm{AW} 2$ & $\mathrm{AW}$ & $\mathrm{N} 1$ & $\mathrm{~N} 2$ \\
\hline Err $_{\mathrm{VCM} 1}$ & 1 & 0 & 0 & 0 & 0 & 0 & 0 & 0 \\
Err $_{\mathrm{ST} 1}$ & 6 & 3 & 8 & 2 & 6 & 4 & 4 & 4 \\
\hline Err $_{\mathrm{VCM} 2}$ & 4 & 1 & 1 & 3 & 4 & 1 & 1 & 0 \\
Err $_{\mathrm{ST} 2}$ & 15 & 14 & 0 & 9 & 3 & 3 & 0 & 0 \\
\hline
\end{tabular}

\subsection{Results}

Skeletons have been generated using the algorithm described by Cornea et al. (2005) with parameters chosen specifically to yield spurious branches. Then, we apply our pruning scheme on the skeletons. In the following, interesting experimental values for the angle threshold have been found to be in the range of 20 degrees to 35 degrees. The optimal threshold depends on the minimum angle spurious branches make with the tube's axis. In practice, since the significance measure is computed for all the points in the volume, users may adjust the threshold on-the-fly and estimate which branches are removed in interactive time. Results on two varying-diameter tubular objects are presented in Fig. 14

The quality of a pruning approach is determined by the number of spurious branches it deletes while preserving the maximum number of relevant branches. Out of the eight volumes in Table 1, only one spurious branch is not deleted. This particular spurious branch is aligned with the tube's axis, thus it cannot be discarded using our method. In addition, spurious branches intersecting with one another and forming a cycle can also be deleted.

Our pruning approach also preserves relevant branches. Only incomplete peripheral branches might get removed, because there is not enough information to estimate the significance measure robustly (see close-up in Fig. 14b). These branches are located in a junction and thus are not suitable to study tubular organs, so their removal does not impede further analysis.

Furthermore, our method is compared to the pruning approach introduced in Serino and Sanniti di Baja (2014). Results in Table 1 show our method is more complete as it deletes more spurious branches than the existing method (see $\operatorname{Err}_{\mathrm{ST} 1}$ and low values for $\operatorname{Err}_{\mathrm{VCM} 1}$ ). Moreover, our method is more sensitive, because it preserves more relevant branches (see $\operatorname{Err}_{\mathrm{VCM} 2}$ and $\operatorname{Err}_{\mathrm{ST} 2}$ ).

\section{Recentering}

Recentering is another post-processing step to improve curve-skeletons. Our recentering approach is also based on orthogonal plane estimation. We handle tubular parts and non-tubular parts, such as junctions, separately.

\subsection{Method}

In a tubular part, new recentered points are defined as the centers of mass of the cross-sections computed at each point of the input non-centered skeleton. Each skeletal branch is processed independently. The recentered skeleton points might be disconnected due to the fact that points are shifted from the input skeleton during the recentering procedure. However, the initial skeleton is connected, which implies that computed orthogonal planes are close from one another. As a result, recentered points remain close (generally at most one point apart from each other). For this reason, each pair of disconnected points is linked by a small digital segment, without altering the centeredness of the skeleton.

\subsection{Junctions}

Junctions are not tubular, thus they must be processed independently. The goal is to decompose a junction into tubular sub-volumes (see Fig. 15b). The junction can be regarded as the division of a 


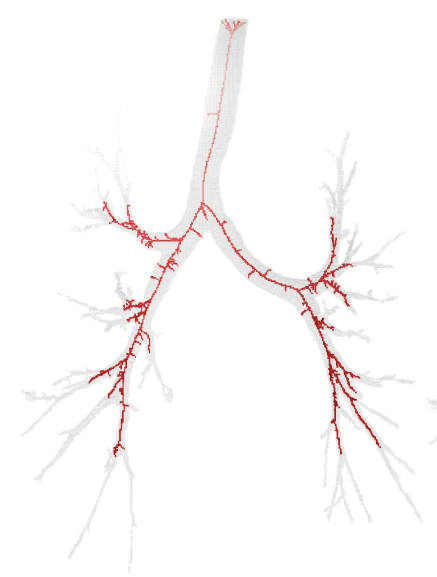

(a)

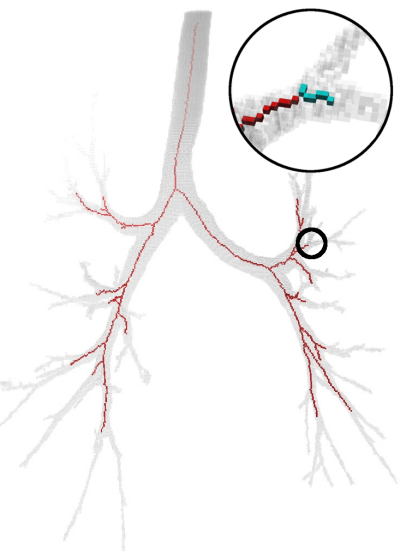

(b)

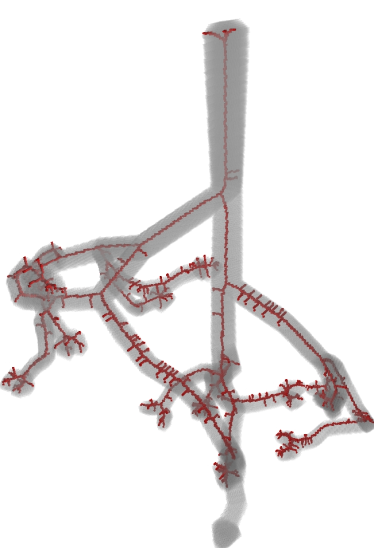

(c)

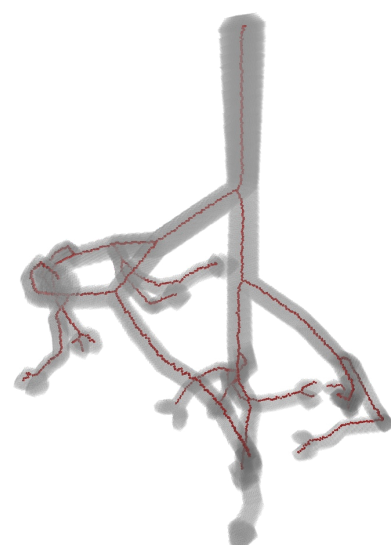

(d)

Figure 14: (a) and (c) Initial skeletons generated using Cornea et al. 2005) on various varying-diameter tubular objects. (b) and (d) Resulting pruned skeletons containing little to no faulty branches. Close-up in Fig. 14b shows examples of deleted relevant branches (in cyan), however, these branches are not complete and not suitable to study the tubular organ.

parent tube into children tubes. Each child should be associated with its parent: the union of a child branch with its parent branch can be seen as a tubular object. Shape decomposition is required to achieve this. In the following, only junctions consisting of three tubes are described for the sake of simplicity.

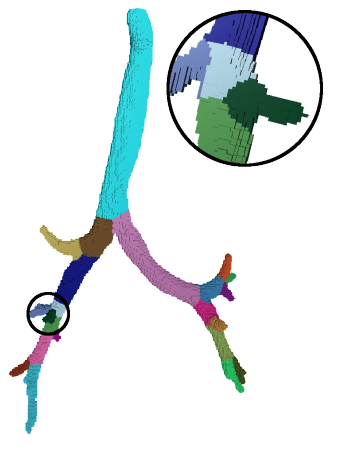

(a)

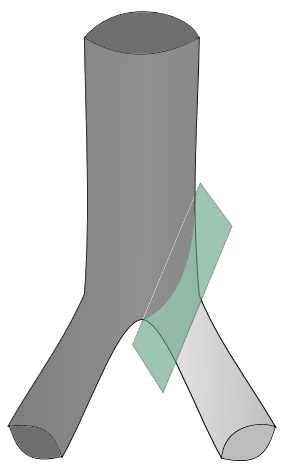

(b)
Figure 15: Methods to create sub-volumes. (a) Shape decomposition obtained using Palágyi et al. (2006). The completeness of the decomposition is dependent on the skeleton. The close-up (black circle) highlights that the decomposition does not always provide tubular parts (see dark green part). (b) Computation of a sub-volume. The cutting plane (in green) isolates the sub-volume, consisting in a parent and a child branch (dark grey), from another child (light grey).

From a given skeleton, we aim at finding the corresponding shape decomposition in order to create sub-volumes. Various state-of-the-art methods ex- ist for this purpose, such as the work presented in Palágyi et al. (2006), which consists in assigning a different label for each branch of the skeleton. Then, the voxels in the volume are given the label of the closest skeletal point. In the context of recentering, the produced cuts do not yield tubular parts (see Fig. 15a). As a result, orthogonal planes cannot be accurately computed with the VCM on the sub-volumes from this decomposition.

Thus, we propose a new shape decomposition scheme. The idea is to find orthogonal planes near a junction which delineate a part from another (see Fig. 15b. These planes are referred to as cutting planes in the following paragraphs.

The input skeleton must possess the same properties as those presented in Section 4.1 (i.e. thin and connected), and have a correspondence with the shape, which means it must be pruned. Shape decomposition is determined by junctions, because they correspond to zones where different parts meet. In a pruned skeleton, branching points are in correspondence with junction areas in the volume, and interesting cutting planes are in the neighborhood of branching points in the skeleton.

Orthogonal planes are used as cutting planes to decompose the shape. Only a few orthogonal planes are interesting for shape decomposition.

For each edge around a branching point $b$, the cutting plane should be positioned at a point where it delineates the tubular branch from the junction area. Orthogonal planes computed near branching points in a child branch intersect the other child 


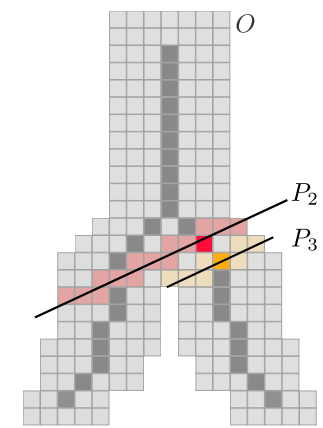

(a)

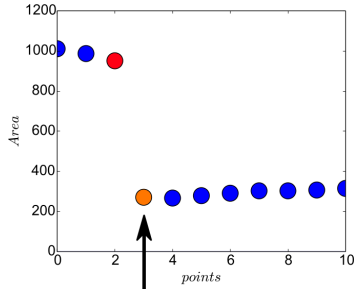

(b)

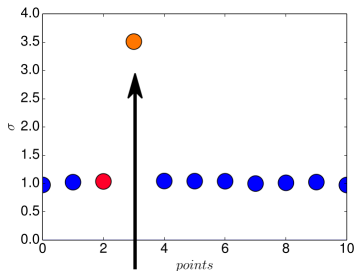

(c)
Figure 16: (a) Computation of orthogonal planes at two points on the same edge. The number of volume points (i.e. the area) in the cross-section varies a lot in junctions. (b) Typical area variation (y-axis) observed along an edge. Points are traversed in an ordered manner from a branching point to the end-point in the skeleton daughter branch. (c) Corresponding $\sigma$ values for the area values of Fig. $16 \mathrm{~b}$. The cutting point is the point where the $\sigma$ value is the highest (shown by an arrow).

branch (see Fig. 16a). In other words, the area of the cross-section is much larger in the junction than in the child branch (see Fig. 16b). The cutting point corresponds to the point where the area variation is the largest (see Fig. 16c). The area $A(p)$ is computed as the number of points in the intersection between the orthogonal plane at $p$ and the volume. To study the area variation, we study the factor $\sigma(x)$ along the edge $E$.

$$
\sigma(x)=\max _{y \in \mathcal{N}_{E}(x)} \frac{A(y)}{A(x)}
$$

where $\mathcal{N}_{E}(x)$ corresponds to the direct backward and forward neighbors of $x$ in the curve $E$.

Then the cutting point $c$ in the edge $E$ is defined as $c=\max _{x \in E} \sigma(x)$. In a junction, only the cutting points of the child branches and their corresponding cutting planes are considered.

The cutting planes provided by the decomposition allow to separate each part but are not proper delineations to create tubular sub-volumes. Indeed, the union of two parts is not tubular in the junction area (see Fig. 17a, in green). The idea is to align the plane with the axis of the other child tube, so as to "extend" the child tube in the parent tube. The direction of the axis is given by the orthogo-

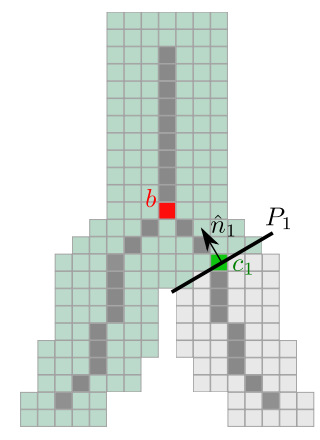

(a)

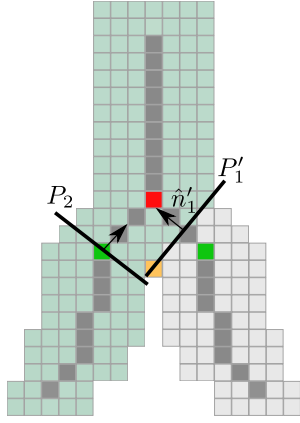

(b)
Figure 17: (a) Sub-volume (in green) computed using orthogonal planes on an input skeleton. Using the decomposition scheme does not allow to recenter skeleton points. (b) Sub-volume (in green) obtained after rotating the plane $P_{1}$ around the center (in orange) so that the new cutting plane $P_{1}^{\prime}$ is orthogonal to the plane $P_{2}$ located in the other child branch.

nal plane normal (i.e. the tangent to the curve) computed in the other child tube. Thus, the first cutting plane $P_{1}$, defined by the normal $\hat{n}_{1}$, is rotated so that it is orthogonal to the other cutting plane $P_{2}$, defined by the normal $\hat{n}_{2}$. The direction of the rotation axis is given as the cross-product of the two normals. Then, the cutting plane normal is $\hat{n}_{1}^{\prime}=\left(\hat{n}_{1} \wedge \hat{n}_{2}\right) \wedge \hat{n}_{2}$. The rotation axis is placed on a point in the cross-section defined by $P_{1}$ and which minimizes the distance to the cross-section defined by $P_{2}$.

After rotation of the planes, two tubular subvolumes $S_{1}$ and $S_{2}$ can be isolated for each branching point $b$ (see Fig. 17b). The skeleton is recentered by computing the centers of mass in each subvolume.

\subsection{Results}

This evaluation aims at verifying whether our recentering scheme produces a recentered skeleton. Tubular volumes with junctions have been generated using a parametric curve. This parametric curve is the theoretical skeleton. Initial noncentered skeletons were generated using the algorithm described in Palágyi and Kuba (1999). Then, we use the Hausdorff distance $d_{H}\left(C, C^{\prime}\right)$ defined as the maximal distance between a point in $C^{\prime}$ and its nearest point in $C$. It allows to measure the dissimilarity between two curves. Here, two Hausdorff distances are of interest: $d_{H}(T, I)$ which is the Hausdorff distance between the theoretical skeleton $T$ and the initial non-centered skeleton 


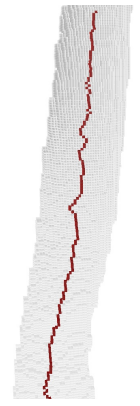

(a)

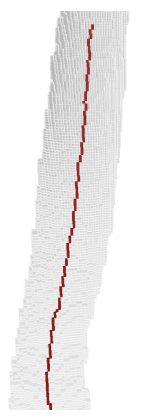

(b)

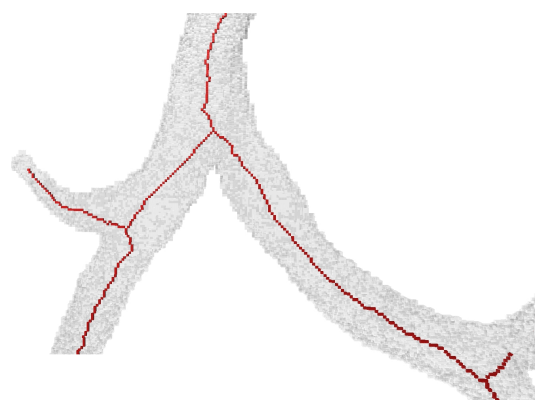

(c)

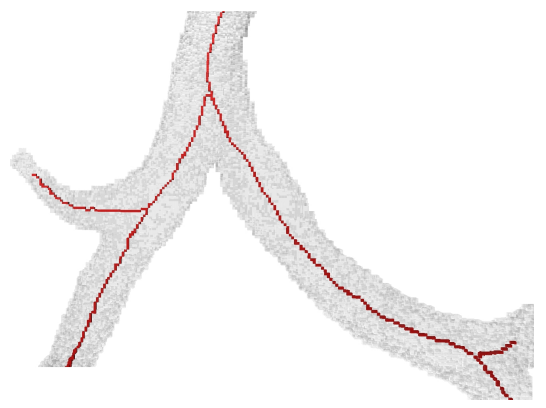

(d)

Figure 18: (a) and (c) Initial non-centered skeletons generated using Palágyi and Kuba (1999) on airway-tree segmentations. (b) and (d) Corresponding resulting recentered skeletons.

Table 2: Hausdorff distances between (middle row) a theoretical centered skeleton $T$ and an initial non-centered skeleton $I$ and (bottom row) between $T$ and a skeleton $I_{R}$ computed by our recentering procedure on various "Y"-shaped volumes.

\begin{tabular}{cccccc}
\hline & $0.45 \pi$ & $0.40 \pi$ & $0.35 \pi$ & $0.30 \pi$ & $0.25 \pi$ \\
\hline$d_{H}(T, I)$ & 3.74 & 2.82 & 3.74 & 4.47 & 5.91 \\
$d_{H}\left(T, I_{R}\right)$ & 1 & 1 & 1 & $\sqrt{2}$ & $\sqrt{2}$ \\
\hline
\end{tabular}

$I$; and $d_{H}\left(T, I_{R}\right)$ which is the Hausdorff distance between the theoretical skeleton and the skeleton $I_{R}$ produced by our recentering method. Volumes are comprised of simple "Y"-junctions consisting in three tubes glued together with varying division angles $(0.45 \pi ; 0.40 \pi ; 0.35 \pi ; 0.30 \pi ; 0.25 \pi)$. The results are presented in Table 2 and show that the recentering procedure produces recentered skeleton from initial non-centered skeleton. Indeed, computed skeletons are at most at $\sqrt{2}$ from theoretical skeletons, which means the theoretical and recentered skeletons points all share a voxel edge. Moreover, our technique is independent on the junction angle.

The recentering procedure was tested on noncentered skeletons generated using the thinning algorithm described in Palágyi and Kuba (1999). Results on real data (airway-tree segmentation) show the skeleton is centered in junctions, and zigzags are properly smoothed (see Fig. 18).

\section{Curve-skeleton computation}

In this section, we show how to use orthogonal planes to compute a curve-skeleton on certain tubular volumes. Tubular parts and junctions are handled in two separate passes in our algorithm.

\subsection{Tracking algorithm}

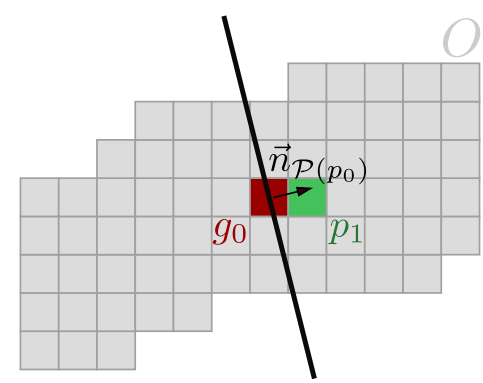

Figure 19: Tracking performed by our algorithm. From a determined center of mass $g_{0}$, we propagate to the next tracked point $p_{1}$ thanks to the plane normal $\vec{n}_{\mathcal{P}\left(p_{0}\right)}$.

The main idea of our algorithm is that the curveskeleton of the object $O$ is the set of centers of mass of the cross-sections defined by the orthogonal planes. The curve-skeleton is obtained by tracking the centers of mass iteratively, as described in our work in Grélard et al. (2016).

Tracking. It is computationally expensive and redundant to go through all points in the volume. A cross-section contains a unique curve-skeleton point so once this point is added to the skeleton, all the remaining points in the cross-section do not need to be processed further. These points are stored in a set $M$ (marked points), and are not used in the remainder of the tracking procedure.

The starting point $p_{0}$ of the algorithm is the point in the volume which has the highest DT value. After the orthogonal plane computation at a point $p_{i}$ we obtain a center of mass $g_{i}$ in the connected component defined by the orthogonal plane and which contains $p_{i}$ (i.e. the cross-section). Then, we prop- 
agate to the next point $p_{i+1}$ in the 26 -neighborhood of $g_{i}$ with the normalized plane normal $\vec{n}_{\mathcal{P}\left(p_{i}\right)}$ :

$$
p_{i+1}=g_{i}+\vec{n}_{\mathcal{P}\left(p_{i}\right)}
$$

until $g_{i}+\vec{n}_{\mathcal{P}\left(p_{i}\right)} \in M$.

Since $g_{i}+\vec{n}_{\mathcal{P}\left(p_{i}\right)} \approx g_{i+1}$ in regular tubular portions of the object, propagation is done either on the next center of mass or on its neighborhood (see Fig. 19). This ensures the algorithm prioritizes points which are well-centered and for which the orthogonal plane normal at $p_{i}$ is close to the plane normal at $p_{i+1}$. This process is done for the two normal directions $\left(g_{i}+\vec{n}_{\mathcal{P}\left(p_{i}\right)}\right.$ and $\left.g_{i}-\vec{n}_{\mathcal{P}\left(p_{i}\right)}\right)$. Once points have been tracked in both directions and the next tracked point is in $M$, then a new point $p_{i+1}$ is chosen as the one with the highest DT value.

In junctions, orthogonal planes are not properly defined. The following paragraphs describe how to specifically detect and handle junctions.

\subsection{Junction detection}

An approach towards junction detection is based on analyzing the local topology of the object, and has been described in Xiong et al. (2012). The authors define the spherical shell intersection (SSI) as the difference between two concentric balls intersected with the volume. The number of connected components of the SSI is equal to two in a regular tube and to the number of involved tubes in a junction (see Fig. 20a). Balls are defined using geodesic distance so as to consider the object locally. The authors show the choice for both the ball radii is crucial to find junctions accurately. Indeed, if the inner ball radius is too small, it might consider a junction area as a regular tube, and if it is too large, it might discard small but relevant protrusions in the volume. The ball radii depend on the local scale of the tube. In Xiong et al. (2012), the authors set the radii empirically based on the distance transform value at the ball center. It makes sense to rely on the distance transform values at centered points for tubes with circular cross-sections because they are equal or close to the tube radius. However, this does not hold for tubes with deformations or elliptical cross-sections. Thus, we propose a way to set both the radii in a manner which makes sense regardless of the shape of the tube. The ball radius is set as the distance between the two farthest points in the cross-section.

All points $p$ satisfying $\operatorname{SSI}(p) \geq 3$ are called 3 shell points and are not added to the skeleton.

\subsection{Junction processing}

At this point, the skeleton is complete in tubes: for each tubular part in the volume, there is an associated part in the skeleton. Each skeletal part (i.e. connected component in the skeleton) must be connected to another in the corresponding junction area. This problem is very close to the one consisting in recentering an existing skeleton in junctions (see Section 5.2). However, skeletal part endpoints might not correspond to cutting points (they are located deeper inside the tube). As a result, if we use the recentering approach, the cutting plane would not be properly positioned, which means the resulting sub-volume would not be tubular and the resulting computed skeleton would not be centered.

Thus, skeleton points in the junction are computed by linking skeletal part endpoints with Bezier curves. First, endpoints must be grouped together if they belong to the same junction area. A junction area $J$ is defined as a maximal connected set of points closer to a 3 -shell point than to a skeleton point (see Fig. 20b). We have to connect the endpoints which belong to the same junction area and which are not terminal points, i.e. for which SSI $>1$.

We define the reference branch as the skeletal branch to which other branches in the same junction must be linked (see Fig. 20c). The reference endpoint $e_{\text {ref }}$ is the point which normal direction is contrary to the most of the other endpoint normals, i.e. for endpoints belonging to the same group $G, e_{\text {ref }}=$ $\arg \max _{e_{1} \in G}\left(\operatorname{card}\left(\left\{e_{2} \in G \mid \vec{n}_{e_{1}} \cdot \vec{n}_{e_{2}}<0\right\}\right)\right)$.

Finally, we link each point $e$ belonging to a same group $G$ to $e_{\text {ref }}$ using Bezier curves. The two control points in the Bezier curve are defined by the orthogonal plane normals at both endpoints. It is also possible to use our recentering procedure after having linked endpoints with Bezier curves to improve the skeleton in junctions if necessary.

All the Bezier curves meet at the reference endpoint, so they are post-processed so as to avoid creating thick parts. For a junction, all voxels of the different Bezier curves are added in parallel, from the endpoint $e \neq e_{\text {ref }}$. Once the Bezier curves intersect, a new common Bezier curve is computed, starting from the intersection point and ending at $e_{\text {ref. }}$ This ensures thinness in junctions.

\subsection{Results}

Our method's efficiency is evaluated by comparison to ground-truth curve-skeletons and to state-of- 


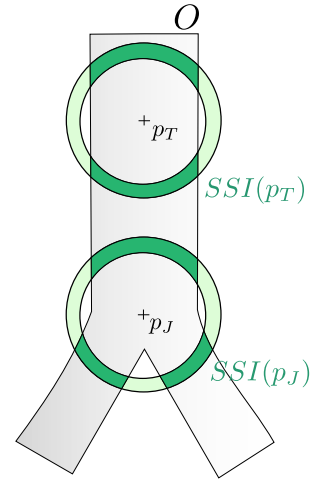

(a)

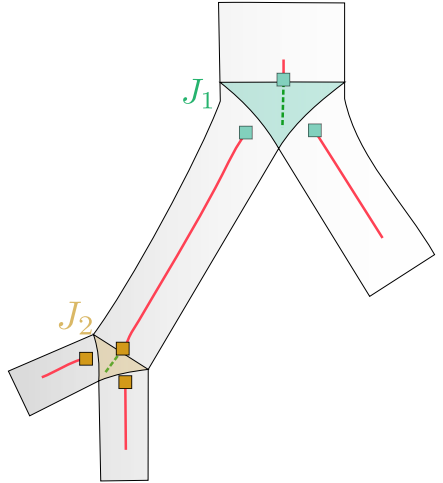

(b)

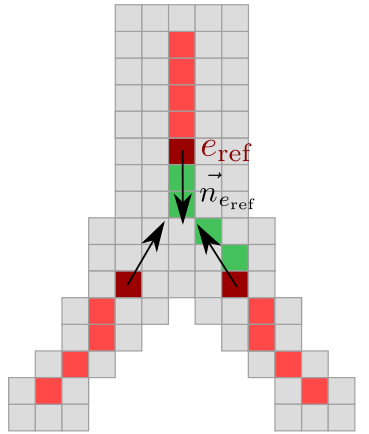

(c)

Figure 20: Junction detection and junction processing. (a) Spherical shell intersection (SSI, in dark green) allow to detect junctions. The number of connected components of the shells is equal to two in a tubular part $\left(p_{T}\right)$ and to three in a junction $\left(p_{J}\right)$. (b) Method to group endpoints from skeletal parts (in red) in junctions in order to link them. Junction areas $J_{1}, J_{2}$ are groups of points which are closer to a 3-shell point (squares) than to a skeletal point (red lines). We group skeletal part endpoints together based on their distance to a junction area. (c) Determining the reference endpoint $e_{\text {ref }}$ to which other endpoints must be linked is done by comparison of the direction of the normal with the other endpoints' orthogonal plane normals. The normals also allow to determine the position of the control points (not shown) and trace the Bezier curve (in green). Here, only one endpoint is linked to the reference endpoint for sake of clarity.

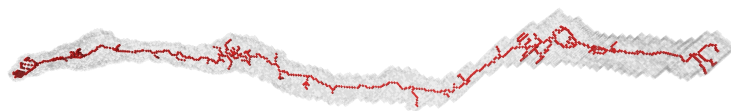

(a)

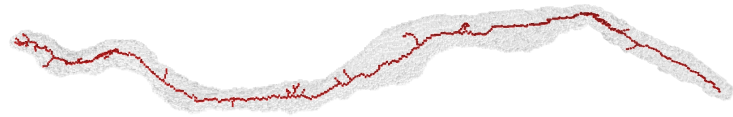

(c)

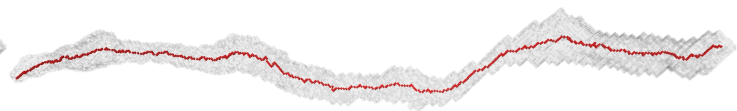

(b)

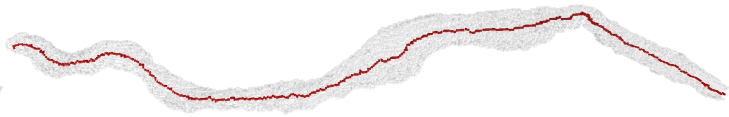

(d)

Figure 21: Skeletons (in red) on neurite volumes (in gray) from the dataset of the SNEMI3D challenge (Kasthuri et al. 2015). On the left, (Figs. (a) and (c)) the skeleton is computed by the thinning method of Couprie et al. (2007). On the right, (Figs. (b) and (d)) the skeleton is computed by our method. Our method is not sensitive to irregularities and major diameter variation. Our skeletons contain no faulty branches.

the-art methods namely euclidean skeleton thinning approach (Couprie et al., 2007) and the potential field method (Cornea et al. 2005) presented in Section 2. Although the limits of both these methods have been discussed, they satisfy interesting properties and are commonly used in our field of application. Our method is tested on both synthetic and real data.

\subsubsection{Synthetic data}

Various simple tubular volumes have been generated using a parametric curve: a cylinder, a cylinder with varying-diameter, referred to as deformed cylinder, a junction, and a curved tube (see Table 3). Four noisy versions of these tubes were generated as well using a simplified version of Kanungo's method (see Section 3.4. For each volume, the expected curve-skeleton $T$ is the parametric curve used to generate the volume. The expected skeleton is compared to the computed skeletons $C$ using the Hausdorff distance $d_{H}$. The skeleton is centered if $d_{H}(T, C) \leq \sqrt{3}$, that is to say if the points in the computed skeleton are neighbors of the points in the expected skeleton. Otherwise, the skeleton is thick or contains faulty branches. Results are shown in Table 3 .

Various parameters for the state-of-the art methods are tested in order to obtain the lowest number of faulty branches and the most complete skeleton. Only results with the best parameters are shown here.

For all non-noisy volumes, all methods produce centered skeletons $\left(d_{H}(T, C) \leq \sqrt{3}\right.$, results not 
Table 3: Hausdorff distance for each method (Thin Couprie et al. 2007), PF (Cornea et al., 2005) and ours) on different noisy volumes.

\begin{tabular}{|c|c|c|c|}
\hline Data & Thin & PF & Ours \\
\hline $\begin{array}{c}\text { Cylinder } \\
\text { Deformed cylinder }\end{array}$ & 9.43 & $\sqrt{2}$ & 1 \\
\hline \begin{tabular}{c} 
Curved tube \\
\hline Junction
\end{tabular} & 9.06 & $\sqrt{3}$ & $\sqrt{2}$ \\
\hline
\end{tabular}

shown).

Regarding noisy volumes, our method produces a skeleton which is the closest to the initial skeleton. The thinning method of Couprie et al. (2007) produces faulty branches which cannot be deleted regardless of the parameter values. As a result, the Hausdorff distance for these skeletons is large. Our method produces results which are close to those of the potential field method of Cornea et al. (2005). Indeed, skeletons produced by both methods are centered. A visual inspection on the junction volume shows our skeleton contains less irregularities than the potential field skeleton and that it is more complete (i.e. there are skeletal points at the extremities of the volume). Moreover, it is not sensitive to irregularities and to major diameter variation. Results for the various methods on synthetic data are available in the online appendix (F. Grélard et al. 2017).

\subsubsection{Real data}

Our method was designed to work on tubular organs. We applied it applied on neurites, airwaytrees and arteries.

Figure 21 shows the results on various volumes of neurites. Compared to the state-of-theart method, our skeleton is thin and produces no faulty branches. Figures 22a, and 22b show the resulting skeletons on two different airway-trees. Figure 22c shows resulting skeletons on pelvic arteries, which also contain junctions. A general visual inspection shows the skeleton does not have the defects of the state-of-the-art methods illustrated in Section 2. Close-ups show the resulting skeleton can capture local information in junctions, as well as in small branches and be exempt of faulty branches throughout the majority of the volume. Moreover, our skeleton is well centered and does not suffer from major irregularities on the surface (see Fig. 22b). In addition to the fact the skeleton is obtained automatically, these properties are interesting in light of the various applications mentioned in Section 1. More results are available online showing our method performs better than state-of-theart methods (F. Grélard et al., 2017). One limit of our approach is that it does not work for organs such as the colon which can be locally non-tubular, as already explained in Section 3.4 .2 .

\section{Conclusion and prospects}

In this article, we have presented several new and original approaches for tubular organ analysis. We presented a method to estimate orthogonal planes is more accurate than state-of-the-art methods and is not sensitive to irregularities on the surface. Orthogonal planes can not only be estimated from a curve, but also directly from a set of voxels. This has helped defining new tools for the study of tubular organs. All these tools, along with the source code, are available online ${ }^{1}$. We presented an automatic pruning method removes close to all the spurious branches, and the associated significance measure is not dependent on the local scale of the shape, which makes it suitable for varying-diameter tubular organs. Pruned skeletons are recentered, which makes virtual endoscopy and geometrical measurements more accurate. Finally, we use the centers of mass of the cross-sections to compute our own curve-skeleton. The resulting skeleton is centered, thin and complete, which is critical to capture the shape of varying-diameter tubes aptly. These methods could be used in an automated workflow in order to make geometrical measurements on a large tubular organ database. The results could eventually help establish the relationship between the structure of the organ and pathologies, and better understand its physiology.

\section{Acknowledgements}

This work received the financial support from the Laboratory of Excellence ANR-10-LABX-57 (TRAIL). 


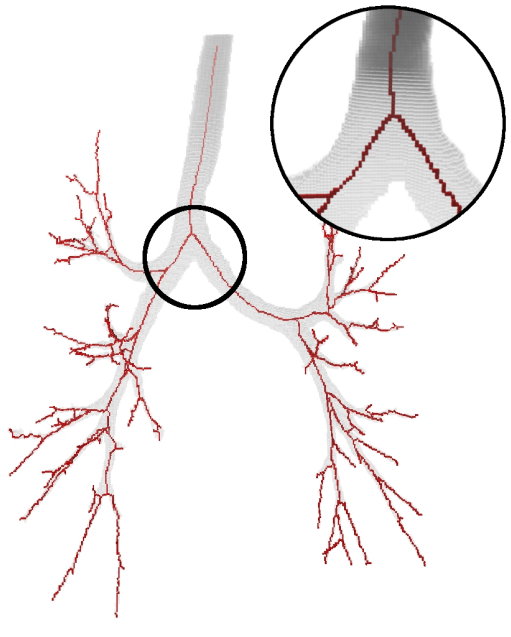

(a)

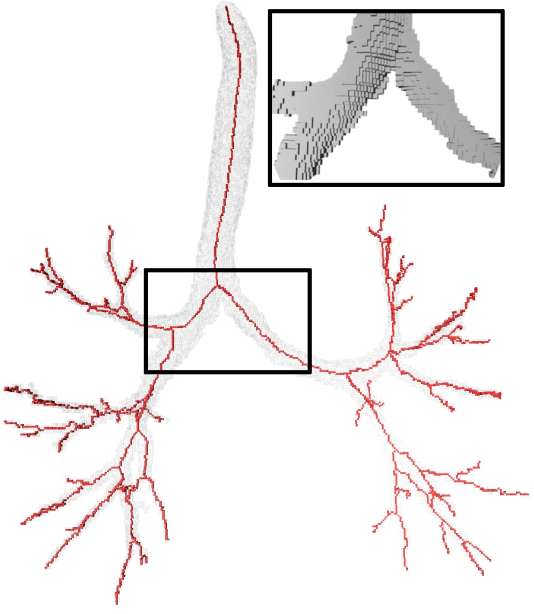

(b)

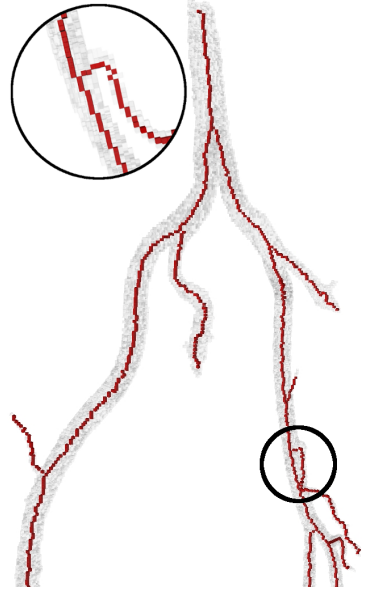

(c)

Figure 22: (a) (b) Centerline (in red) extraction on two different airway tree volumes (in gray) and (c) pelvic arteries acquired in MRA. The volume for the pelvis arteries is cropped due to the large image size. Fig. 22a corresponds to the Fig. 2 in Section 2 for comparison with state-of-the-art methods. The skeleton is complete, that is to say there is a branch in the skeleton for each bronchus in the volume. This is true even in cases where bronchi have low diameter (cases where only the skeleton in red is visible). Moreover, junctions are properly processed through the second pass of our algorithm. Our skeleton is not sensitive to irregularities on the surface of the volume (see close-up on the volume in Fig. $22 \mathrm{~b}$ ).

\section{References}

Arcelli, C., Sanniti di Baja, G., 1993. Euclidean skeleton via centre-of-maximal-disc extraction. Image and Vision Computing 11 (3), $163-173$.

Barbieri, S., Meloni, P., Usai, F., et al., 2015. Skeleton Lab: an Interactive Tool to Create, Edit, and Repair CurveSkeletons. In: STAG. The Eurographics Association.

Bauer, C., Bischof, H., 2008. Extracting curve skeletons from gray value images for virtual endoscopy. In: MIAR. Lecture Notes in Computer Science. Springer.

Bradley, R. S., Withers, P. J., 2016. Post-processing techniques for making reliable measurements from curveskeletons. Computers in Biology and Medicine 72, 120 131.

Cornea, N. D., Silver, D., Min, P., 2007. Curve-skeleton properties, applications, and algorithms. IEEE Trans. Vis. Comput. Graph., 530-548.

Cornea, N. D., Silver, D., Yuan, X., et al., 2005. Computing hierarchical curve-skeletons of 3D objects. The Visual Computer.

Couprie, M., Coeurjolly, D., Zrour, R., Oct. 2007. Discrete bisector function and Euclidean skeleton in 2D and 3D. Image and Vision Computing 25 (10), 1519-1698.

Cuel, L., Lachaud, J.-O., Mérigot, Q., et al., 2015. Robust geometry estimation using the generalized voronoi covariance measure. SIAM Journal on Imaging Sciences 8 (2), 1293-1314.

Cuel, L., Lachaud, J.-O., et al., 2014. Voronoi-based geometry estimator for 3D digital surfaces. In: DGCI. Springer, pp. $134-149$

D. Coeurjolly, J-O Lachaud, B. K., et al., 2016. DGtal: Digital geometry tools and algorithms library. http://dgtal. org
Dima, A., 2002. Computer aided image segmentation and graph construction of nerve cells from $3 \mathrm{~d}$ confocal microscopy scans. Ph.D. thesis, TU Berlin.

F. Grélard, F. Baldacci, A. V., et al., 2017. Technical report. https://fgrelard.github.io/ OrthogonalPlaneBasedTools/

Flasque, N., Desvignes, M., Constans, J.-M., Revenu, M., 2001. Acquisition, segmentation and tracking of the cerebral vascular tree on $3 \mathrm{~d}$ magnetic resonance angiography images. Medical Image Analysis 5 (3), 173-183.

Grélard, F., Baldacci, F., Vialard, A., et al., 2015. Precise cross-section estimation on tubular organs. In: CAIP. pp. 277-288.

Grélard, F., Baldacci, F., Vialard, A., et al., 2016. Centerlines of tubular volumes based on orthogonal plane estimation. In: DGCI 2016. pp. 427-438.

Hassouna, M. S., Farag, A. A., 2009. Variational curve skeletons using gradient vector flow. IEEE Trans. on Pattern Anal. and Mach. Intell. 31 (12), 2257-2274.

Janoos, F., Mosaliganti, K., Xu, X., et al., 2009. Robust $3 \mathrm{~d}$ reconstruction and identification of dendritic spines from optical microscopy imaging. Medical Image Analysis 13 (1), $167-179$.

Kang, D.-G., Seong, W., Ra, J. B., Mar. 2006. Vessel crosssection determination based on nonrigid registration and electric field model. In: Manduca, A., Amini, A. A. (Eds.), Medical Imaging 2006: Physiology, Function, and Structure from Medical Images. Vol. 6143 of Proceedings SPIE. pp. $764-771$.

Kanungo, T., Haralick, R., Baird, H., et al., 2000. A statistical, nonparametric methodology for document degradation model validation. IEEE Trans. Pattern Anal. and Mach. Intell. 22 (11), 1209-1223.

Kasthuri, N., Hayworth, K. J., Berger, D. R., et al., 2015. 
Saturated reconstruction of a volume of neocortex. Cell 162 (3), 648-661.

Lang, S., Muller, B., Dominietto, M. D., et al., 2012. Three-dimensional quantification of capillary networks in healthy and cancerous tissues of two mice. Microvascular Research 84 (3), $314-322$.

Lee, T.-C., Kashyap, R. L., Chu, C.-N., 1994. Building skeleton models via 3 -d medial surface/axis thinning algorithms. CVGIP: Graph. Models Image Process. 56 (6), 462-478.

Lo, P., Sporring, J., Ashraf, H., Pedersen, J. J., de Bruijne, M., 2010. Vessel-guided airway tree segmentation: A voxel classification approach. Medical Image Analysis 14 (4), $527-538$.

Members, W. G., Rosamond, W., Flegal, K., Furie, K., Go, A., et al., 2008. Heart disease and stroke statistics : 2008 update: A report from the american heart association statistics committee and stroke statistics subcommittee. Circulation 117 (4), e25-e146.

Mérigot, Q., Ovsjanikov, M., Guibas, L., 2011. Voronoibased curvature and feature estimation from point clouds. Visualization and CG 17 (6), 743-756.

Murray, C. J. L., Lopez, A. D., Nov. 1996. Evidence-Based Health Policy-Lessons from the Global Burden of Disease Study. Science 274 (5288), 740-743.

Palágyi, K., Kuba, A., 1999. Directional 3d thinning using 8 subiterations. In: DGCI'99. Springer Berlin Heidelberg, pp. 325-336.

Palágyi, K., Tschirren, J., Hoffman, E., Sonka, M., 2006. Quantitative analysis of pulmonary airway tree structures. Computers in biology and medicine 36 (9), 974-996.

Postolski, M., Janaszewski, M., Kenmochi, Y., Lachaud, J.O., 2012. Tangent estimation along $3 \mathrm{~d}$ digital curves. In: ICPR. pp. 2079-2082.

Serino, L., Sanniti di Baja, G., Aug 2014. Pruning the 3d curve skeleton. In: Pattern Recognition (ICPR), 2014. pp. 2269-2274.

Shaked, D., Bruckstein, A. M., Feb. 1998. Pruning medial axes. Comput. Vis. Image Underst. 69 (2), 156-169.

Sobiecki, A., Jalba, A., Telea, A., 2014. Comparison of curve and surface skeletonization methods for voxel shapes. Pattern Recognition Letters 47, 147-156.

Tagliasacchi, A., Delame, T., Spagnuolo, M., et al., 2016. 3D Skeletons: A State-of-the-Art Report. Computer Graphics Forum.

Tschirren, J., Hoffman, E. A., McLennan, G., et al., 2005. Intrathoracic airway trees: segmentation and airway morphology analysis from low-dose ct scans. Medical Imaging, IEEE Transactions on 24 (12), 1529-1539.

Xiong, G., Chen, C., Chen, J., Xie, Y., Xing, L., 2012. Tracking the motion trajectories of junction structures in $4 \mathrm{~d}$ ct images of the lung. Physics in Medicine and Biology 57 (15), 4905. 